\title{
New physics in the third generation quark sector: LHC predictions from LEP and Tevatron anomalies
}

\author{
Grégory MOREAU* \\ Laboratoire de Physique Théorique \\ CNRS and Université Paris-Sud 11, Bât. 210 \\ F-91405 Orsay Cedex, FRANCE \\ E-mail: moreaulth.u-psud.fr
}

\begin{abstract}
Within the paradigm of warped extra dimensions, third generation quarks are expected to be the most sensitive to effects beyond the Standard Model. The anomalies observed at the LEP and Tevatron colliders in the forward-backward asymmetries of the bottom $\left(A_{\mathrm{FB}}^{b}\right)$ and top $\left(A_{\mathrm{FB}}^{t}\right)$ quarks can thus be seen as early signatures of warped extra-dimensional scenarios. We propose a realization of such a scenario, with a gauge custodial symmetry in the bulk, which allows to address simultaneously the $A_{\mathrm{FB}}^{b}$ anomaly and the discrepancies observed recently on $A_{\mathrm{FB}}^{t}$ at high top quark rapidities and $t \bar{t}$ invariant masses. We then show that the various phenomenological constraints arising from LEP, Tevatron and LHC can be satisfied within the considered model. The model predicts new features, induced by a Kaluza-Klein excitation of the gluon at a mass $\sim 1.5-2 \mathrm{TeV}$, in top quark pair production at the $\sqrt{s}=7 \mathrm{TeV}$ LHC.
\end{abstract}

The 2011 Europhysics Conference on High Energy Physics-HEP 2011,

July 21-27, 2011

Grenoble, Rhône-Alpes France

${ }^{*}$ Speaker. 


\section{Introduction}

There is increasing evidence that departures from the Standard Model (SM) are experimentally observed in the sector of third generation quarks. First, there is the longstanding anomaly of the forward-backward (FB) asymmetry for $b$-quark jets, $A_{\mathrm{FB}}^{b}$, measured at LEP which differs by almost three standard deviations from the SM value at the $Z$ boson pole. Then, the D0 [1] and CDF [2] collaborations have reported results on the FB asymmetry, $A_{\mathrm{FB}}^{t}$, in top quark pairs produced at the Tevatron collider that are significantly higher than the SM expectation. More recently, this excess has been confirmed by updated CDF data based on an higher luminosity [3] which, interestingly, show that the excess in the $t \bar{t}$ rest frame appears mainly at high $t \bar{t}$ invariant masses $\left(M_{t \bar{t}}\right)$ - being at +3.4 standard deviations from the SM value above $M_{t \bar{t}}=450 \mathrm{GeV}-$ as well as at high rapidities $(\Delta y)$ - being at +1.9 standard deviations from the SM for $|\Delta y|>1$.

From a theoretical point of view, over the past decade, there have been intensive developments about an attractive alternative to supersymmetry : the warped extra-dimension theory proposed by Randall and Sundrum (RS) [4]. This context allows to address the $A_{\mathrm{FB}}^{b}$ anomaly at LEP [6]. The KK gluon exchange in the s-channel can also soften [7] the discrepancy between the value of $A_{\mathrm{FB}}^{t}$ measured at the Tevatron [2] and its SM value. Here, we pursue our earlier efforts to explain these anomalies on $A_{\mathrm{FB}}^{b}[6]$ and $A_{\mathrm{FB}}^{t}$.

\section{The theoretical model}

To protect the EW observables while allowing for not too heavy KK gauge bosons, $M_{K K} \sim 1.5-$ $2 \mathrm{TeV}$, we consider the bulk gauge custodial symmetry, $\mathrm{SU}(2)_{\mathrm{L}} \times \mathrm{SU}(2)_{\mathrm{R}} \times \mathrm{U}(1)_{\mathrm{X}}$ [8]. The chiral quarks are promoted to the following universal representations under this symmetry group, looking e.g. at the third generation, one has,

$$
q_{1 L} \in\left(\begin{array}{ccc}
t_{1 L} & b_{L}^{\prime} & q_{-4 / 3 L}^{\prime} \\
b_{1 L} & q_{-4 / 3 L}^{\prime \prime} & q_{-7 / 3 L}^{\prime}
\end{array}\right)_{-5 / 6} q_{2 L} \in\left(\begin{array}{cc}
q_{5 / 3 L}^{\prime} & t_{2 L} \\
t_{L}^{\prime} & b_{2 L}
\end{array}\right)_{2 / 3} b_{R} \in\left(b_{R} q_{-4 / 3 R}^{\prime}\right)_{-5 / 6} \quad t_{R} \in\left(t_{R}\right)_{2 / 3}
$$

The $q_{1 L}$ and $q_{2 L}$ multiplets mix together on the Planck-brane resulting in the SM doublet $Q_{L}$ mainly composed here by the $q_{2 L}$ component. The parameters $c_{f}$ fixing the 5-dimensional masses for each fermion $f, \pm c_{f} k, 1 / k$ being the $A d S$ curvature radius, are : $c_{u_{L}}=c_{d_{L}} \simeq 0.44, c_{u_{R}}, c_{d_{R}} \simeq 0.80, c_{c_{L}}=$ $c_{s_{L}} \simeq 0.62, c_{c_{R}} \simeq 0.62, c_{s_{R}} \simeq 0.49, c_{t_{L}}=c_{b_{L}} \simeq 0.51, c_{t_{R}} \simeq-1.30, c_{b_{R}} \simeq 0.53$. These $c$ values also generate the correct order of magnitude for the fermion masses.

\section{Top asymmetries at Tevatron}

The parameters lead to the axial quark couplings, $a_{q} \simeq-0.41$ and $a_{t} \simeq 3.41$, and to the low mass, $M_{K K}=1.5 \mathrm{TeV}$, maximizing the RS contribution to the FB asymmetry for the top quark at the Tevatron. The left-hand side of Figure 1 shows that the RS contribution increases the whole FB asymmetry, leading to a much better agreement with the recent unfolded CDF results than in the SM. The remaining discrepancy of $\sim 1.7 \sigma$ could be attributed to either a statistical fluctuation or higher order EW and QCD corrections. 

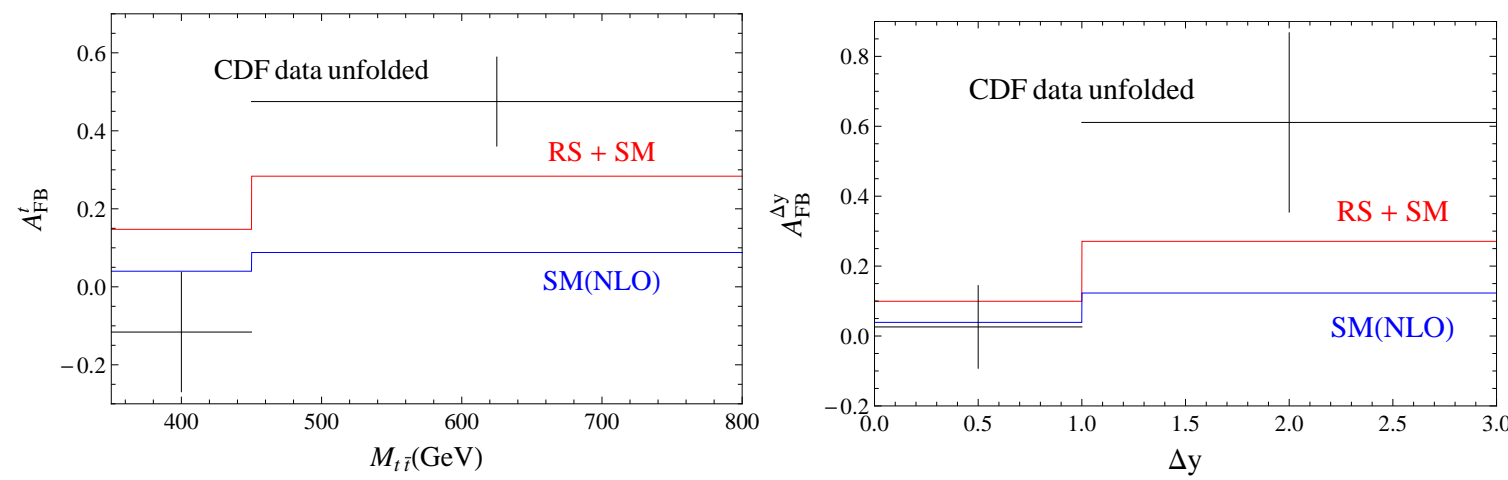

Figure 1: Left: The full top quark asymmetry integrated in the two energy ranges $[350,450]$ and $[450,900]$ of invariant mass $M_{t \bar{t}}$ (in $\mathrm{GeV}$ ) computed within the RS extension of the SM, $A_{\mathrm{FB}}^{t}$, with $\mu_{\mathrm{F}}=\mu_{\mathrm{R}}=m_{t}=$ $172.5 \mathrm{GeV}$ [red lines] and compared to the $\mathrm{SM}$ prediction at $\mathrm{NLO}, A_{\mathrm{FB}}^{\mathrm{SM}}$ [blue lines] as well as to the unfolded $\mathrm{CDF}$ data for $m_{t}=172.5 \mathrm{GeV}$ [black crosses for experimental errors]. Right: The asymmetries $A_{\mathrm{FB}}^{|\Delta y|<1}$ and $A_{\mathrm{FB}}^{|\Delta y|>1}$ computed in the RS extension [red lines] and compared to the SM prediction at NLO [blue lines] as well as to the unfolded CDF data [black crosses].

The fit on the asymmetry $A_{\mathrm{FB}}^{t}$ integrated over the whole $M_{t \bar{t}}$ range is also greatly improved in our RS scenario compared to the SM case, as one sees by comparing the theoretical prediction of our RS extension with the measurement [for $\mu_{\mathrm{R}}=\mu_{\mathrm{F}}=m_{t}=172.5 \mathrm{GeV}$ ] - Tevatron data [3]: $0.158 \pm 0.075 ; \mathbf{S M}[\mathbf{N L O}][3]: 0.058 \pm 0.009(-1.33 \sigma)$; RS+SM: $\quad 0.189 \pm 0.010(+0.42 \sigma)$ where the standard deviations of the central theoretical values relatively to the experimental value are given in brackets.

The FB asymmetries at low $(|\Delta y|<1)$ and high $(|\Delta y|>1)$ top rapidities, $y_{t}=\Delta y / 2$, have been measured by the CDF collaboration [3] with a rapidity cut $|\Delta y|<3$. The right-hand side of Figure 1, in which are given these unfolded results, illustrates that the fit to data is improved in the RS realization compared to the SM situation.

\section{Bottom asymmetry at LEP}

With the choice of Eq. 2.1 of representations and our $c$ values, the anomaly on $A_{\mathrm{FB}}^{b}$ can be cured, while keeping $R_{b}$ in good agreement with the LEP data. The obtained deviations with respect to data are given in Table 1 , for a $Z^{\prime}$ coupling $g_{Z^{\prime}} \simeq 2$.6. In Table 1 , we also present the list of EW precision observables in the quark sector in the SM and RS cases for $M_{K K}=1.5 \mathrm{TeV}$. We see that within RS, each observable is in a good agreement with its measurement.

\begin{tabular}{|c|c|c|c|c|c|c|c|c|c|c|}
\hline Obs. & $A_{\mathrm{FB}}^{b}$ & $R_{b}$ & $A_{\mathrm{FB}}^{c}$ & $R_{c}$ & $A_{\mathrm{FB}}^{s}$ & $\Gamma_{\text {had }}^{Z}$ & $\Gamma_{\text {tot }}^{W}$ & $\left\langle Q_{\mathrm{FB}}\right\rangle$ & $C_{1 u}+C_{1 d}$ & $C_{1 u}-C_{1 d}$ \\
\hline SM & $2.7 \sigma$ & $0.8 \sigma$ & $0.9 \sigma$ & $0.0 \sigma$ & $0.6 \sigma$ & $1.3 \sigma$ & $0.2 \sigma$ & $1.1 \sigma$ & $0.2 \sigma$ & $1.1 \sigma$ \\
\hline RS & $1.2 \sigma$ & $1.2 \sigma$ & $0.9 \sigma$ & $0.5 \sigma$ & $0.2 \sigma$ & $1.0 \sigma$ & $0.2 \sigma$ & $0.1 \sigma$ & $0.8 \sigma$ & $0.1 \sigma$ \\
\hline
\end{tabular}

Table 1: List of EW precision observables in the quark sector with their standard deviations [in absolute value] for the theoretical predictions with respect to experimental data in the SM and in our RS realization. 

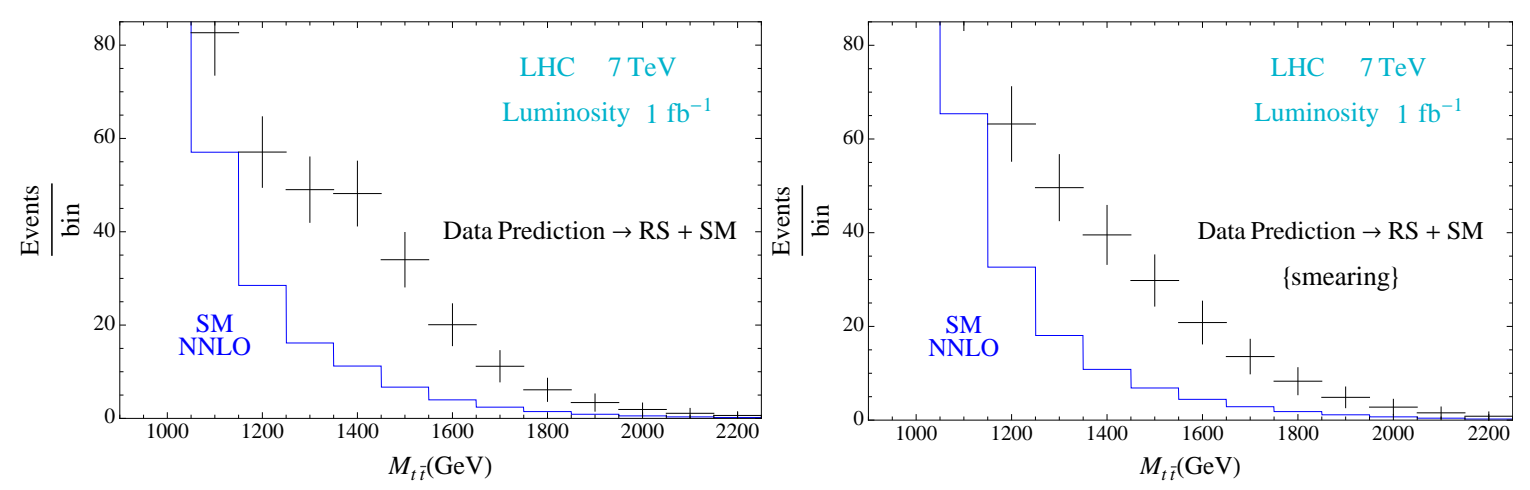

Figure 2: The distributions of the invariant mass $M_{t \bar{t}}$ (in $\mathrm{GeV}$ ) at the $\mathrm{LHC}$ assuming $100 \mathrm{GeV}$ bins with a luminosity of $\mathscr{L}=1 \mathrm{fb}^{-1}$. The $\mathrm{SM}$ at approximate $\mathrm{NNLO}\left(\mu_{\mathrm{F}}=\mu_{\mathrm{R}}=m_{t}=173 \mathrm{GeV}\right)$ [blue histogram] is shown together with the RS contribution [in black]. The effect of smearing is only implemented in the right-hand side figure.

\section{Predictions for $\mathrm{LHC}$}

The present RS realization, which resolves the $A_{\mathrm{FB}}^{b}$ and $A_{\mathrm{FB}}^{t}$ anomalies, would lead to striking effects in the $M_{t \bar{t}}$ distribution that can be observed at the $\sqrt{s}=7 \mathrm{TeV}$ LHC with the expected luminosity of a few $\mathrm{fb}^{-1}$. These are an excess of events due to the large $g^{(1)} \bar{t}_{R} t_{R}$ coupling and a "peak" effect due to the KK gluon resonance, as shown in the left-hand side of Figure 2. Displayed are the $M_{t \bar{t}}$ distributions in the SM and the RS scenario with the bands indicating the statistical error. In the right-hand side of Figure 2, we have taken into account the fact that there is a finite experimental resolution in the measurement of the invariant mass $M_{t \bar{t}}$.

In conclusion, we have presented a RS scenario which allows for a common explanation of the anomalies observed at LEP and Tevatron. Such a scenario can be tested at the LHC with the sample of pair produced top quarks that will be collected this year. This data sample should show a clear excess of events for $t \bar{t}$ invariant masses around $1.5 \mathrm{TeV}$.

\section{References}

[1] D0 Collaboration (V. M. Abazov et al.), Phys. Rev. Lett. 100 (2008) 142002; CDF Collaboration (T. Aaltonen et al.), Phys. Rev. Lett. 101 (2008) 202001.

[2] CDF Collaboration, CDF/ANAL/TOP/PUBLIC/9724, March 2009; http://www-cdf.fnal.gov/physics/new/top/2009/tprop/Afb/.

[3] CDF Collaboration (T. Aaltonen et al.), arXiv:1101.0034 [hep-ex].

[4] L. Randall and R. Sundrum, Phys. Rev. Lett. 83 (1999) 3370.

[5] T. Gherghetta and A. Pomarol, Nucl. Phys. B586 (2000) 141.

[6] A. Djouadi et al., Nucl. Phys. B773 (2007) 43; B797 (2008) 1.

[7] A. Djouadi et al., Phys. Rev. D82 (2010) 071702; Phys. Lett. B701 (2011) 458.

[8] K. Agashe, A. Delgado, M. J. May and R. Sundrum, JHEP 0308 (2003) 050. 